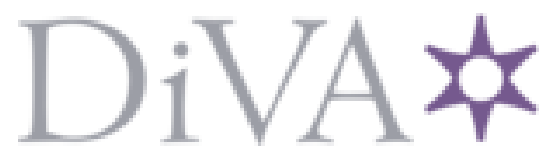

http://www.diva-portal.org

This is the published version of a paper presented at Clawar 2014: 17th International Conference on Climbing and Walking Robots CLAWAR, Poznan, Poland, 21 - 23 July, 2014.

Citation for the original published paper:

Albitar, H., Ananiev, A., Kalaykov, I. (2014)

Stability study of underwater crawling robot on non-horizontal surface.

In: Mobile Service Robotics: Clawar 2014: 17th International Conference on Climbing and

Walking Robots and the Support Technologies for Mobile Machines: Poznan, Poland 21 - 23

July 2014 (pp. 511-519). Singapore: World Scientific

N.B. When citing this work, cite the original published paper.

Permanent link to this version:

http://urn.kb.se/resolve?urn=urn:nbn:se:oru:diva-35985 


\title{
STABILITY STUDY OF UNDERWATER CRAWLING ROBOT ON NON-HORIZONTAL SURFACE
}

\author{
H.Albitar * , A.Ananiev and I.Kalaykov \\ AASS, Örebro University, \\ Örebro, 70182, Sweden \\ *E-mail: houssam.albitar@oru.se \\ www.aass.oru.se
}

\begin{abstract}
This paper introduces a study of a concept of flexible crawling mechanism to design an industrial underwater cleaning robot, which is evaluated from the viewpoint of the capability to work underwater, scanning the desired surface, and bearing the reactions. This can be used as a robotic application in underwater surface cleaning and maintenance. In this study we focused on realizing the adhesion on the surface in stationary and in motion, bearing reactions, enabling the needed locomotion types for scanning, and achieving the stability in different situations on the surface.
\end{abstract}

Keywords: crawling robot; underwater; locomotion; stability

\section{Introduction}

Generally, surface cleaning takes place in 3D-spaces, but we consider the robot operating on a smooth nearly flat local $2 \mathrm{D}$-space around it. Therefore the cleaning robot needs minimum two active (controllable) degrees of freedom (2DOFs) to move. In addition, additional passive DOFs are needed to conform the robot's structure to the concavity/convexity of the surface. We proposed ${ }^{1}$ an in-water crawling robot having four main subsystems, namely: locomotion(crawling, rotation and stepping), adhesion(suction cups), cleaning (pressurized water jet), and neutralization of reaction forces (water jet reaction), details given in a previous publication. ${ }^{1}$

The locomotion subsystem is the basic component providing all movements of the robot. We selected the crawling mechanism because: it adapts to the surface, it can be used for different types of surface material, it is a simple mechanism, and it is stable with low center of gravity and large base support in the cleaning process.

In this work we study the stability of the robot on the surface and the 
effect of the crawling and the inclination angle on keeping the adhesion to the surface in stationary phase (all suction cups are ON, motors are OFF) and in motion phase (two suction cups are OFF, one motor is ON). This analysis helps us to estimate the needed force for each suction cup, as well as to control the balancing force in the case of different types of locomotion when the suction cups are off in order to avoid critical situations.

In Section 2 we briefly describe the proposed robot prototype and its major functions and locomotion. The core of the paper is in Section 3, where two typical options are discussed, namely in stationary phase and in motion. The analysis is to find the minimum required force of vacuum suction cups and force of water jets to keep the robot in contact with the surface continuously. Conclusions highlight the main results.

\section{The in-water cleaning robot and its locomotion}
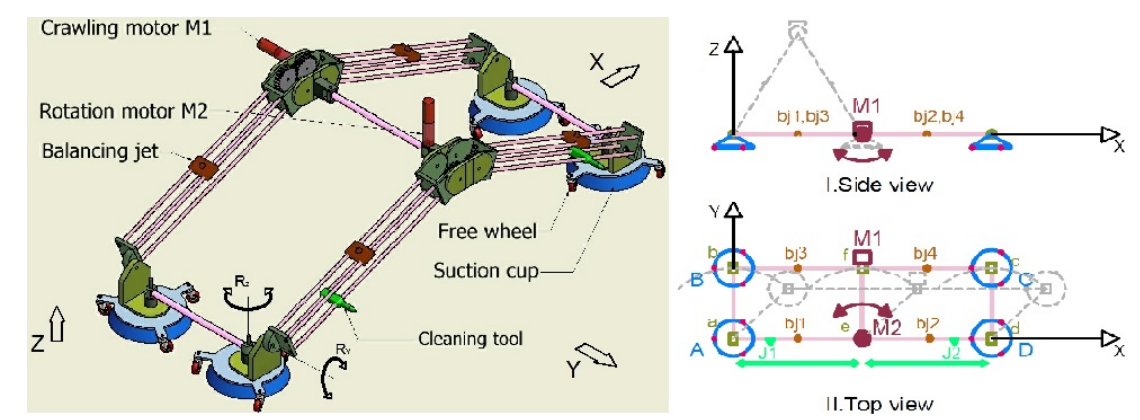

\subsection{Robot concept}

Fig. 1. The crawling robot

The seven links of the robot are connected through six joints (Fig.1), each joint has two rotational DOF $\mathcal{R}_{Y}$ and $\mathcal{R}_{Z}$, two motors are used M1 for crawling and $\mathbf{M} 2$ for rotation and the rest joints are passive in both axes. The robot has a form of two parallel bipeds connected with three parallel links. To materialize the crawling motor $\mathbf{M} 1$ actively controls the angle between the legs to perform desired step size.

At the same time, both bipeds with the connecting links form two parallelograms with three links parallel in all possible configurations. When motor M2 is moving, the rest joints passively follow the rotation.

Four suction cups are attached to the four corner joints, each cup is surrounded by three small free wheels to minimize the friction of movement when any of these cups is not enabled (OFF-state).Due to the limitations of most of the adhesion techniques, we selected adhesion by suction cups for 
their light weight and simple control that allows movement over arbitrary surfaces made of non-ferromagnetic materials. ${ }^{2}$ This type of adhesion is well developed and commercially available.

The cleaning subsystem contains two water jets directed on controllable varying angle to the surface. They are installed on one of biped links. The jets slide on these two links and a separate control system regulates the linear speed and time of cleaning in accordance to the nature of bio-fouling. A vision camera provides feedback to the human operator for the cleaning quality. We do not provide more details in this paper, as its focus is at the robot structure and operation.

In the cleaning subsystem, the force of the water being discharged from the jet nozzle creates an equal and opposite reaction, which makes the nozzle recoil in the opposite direction of the water flow. ${ }^{3-5}$ This effect becomes stronger as water jet flow increases.

The cleaning water jets are directed at certain angle to the surface depending on the needs of the cleaning process. Therefore, the generated reaction force drags the robot away from the surface, for compensating and neutralizing the reaction force four dedicated waters jets are installed on the four links. While robot is in contact to the surface, thrust forces generated by these jets reinforce the adhesion. The water flow through the balancing jets is regulated such that the vector sum of all forces is nullified.

\subsection{Robot locomotion}

The robot moves at any $X-Y$ surface direction by combining the two active DOFs $\mathcal{R}_{Y}$ and $\mathcal{R}_{Z}$, actuated by motors M1 and M2 respectively, with $\mathrm{ON} / \mathrm{OFF}$ activation of the suction cups. Enabled suction at one place and disabled suction at others gives the needed freedom of robot to move by activating M1 and M2 in coordinated way with suction cups' activation. By special control sequences the robot can perform three basic movements:crawling movement (on $X$-axis), changing orientation (rotation around $Z$-axis), and stepping forward (on $Y$-axis). Combining these movements the robot can scan the entire $2 \mathrm{D}$ space to be cleaned.

Crawling (on X-axis) is achieved by the pair of biped links, it is assumed the bipeds are at max-span pose (angle between the legs is $180^{\circ}$ ) at initial position. The moving straight forward starts with switching OFF the rear pair of suction cups, and starting the crawling motor M1 to initiate contracting motion of both bipeds. After desired angle is achieved, M1 is stopped and the suction cups are switched ON again. The expanding motion is done by switching OFF the front cups, then M1 rotates in the opposite 

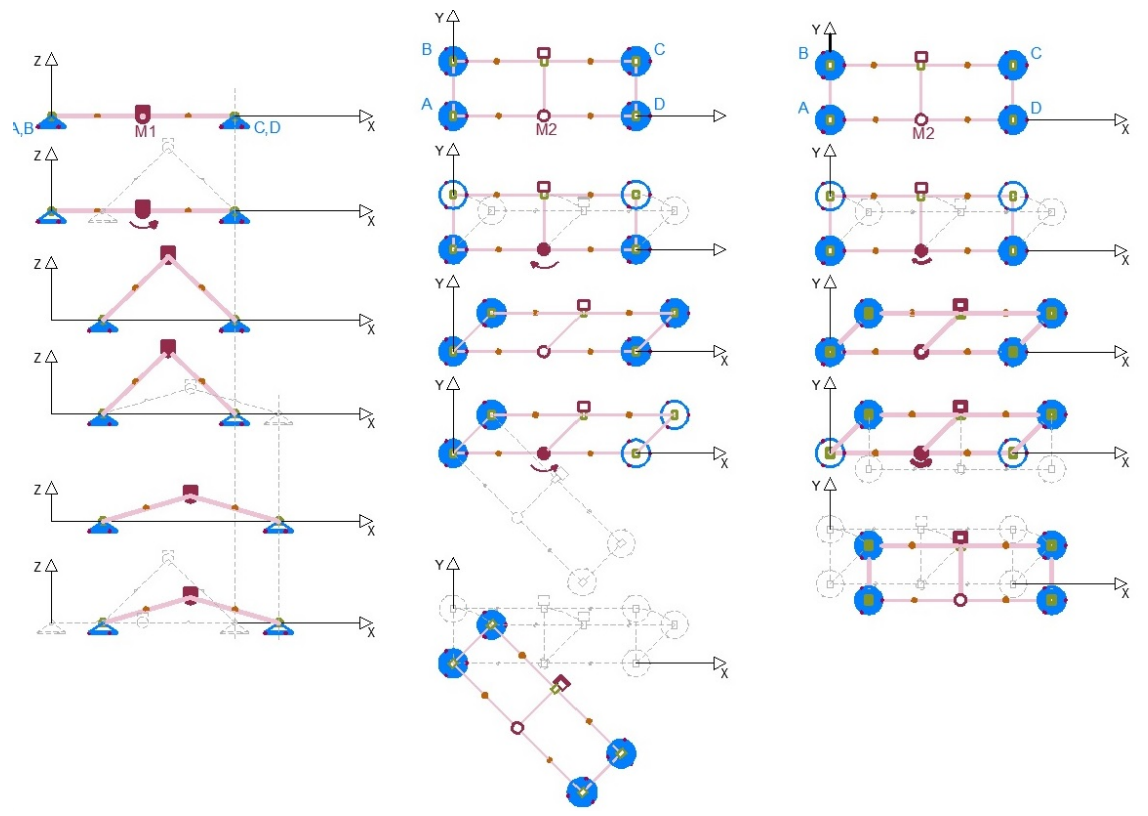

Fig. 2. Possible robot locomotion types: crawling, rotation, stepping forward

direction. After a desired angle is achieved, $\mathbf{M 1}$ is stopped, and the suction cups are switched ON again, the robot is fixed at the final position (Fig.2).

During the described movement, when any of the suction cups is OFF, there is a need to keep the robot in contact with the surface because the suction cups have passive DOF around $\mathcal{R}_{Y}$. Therefore, in the course of motion neutralizing (balancing) water jets are activated. For maximum production rate, the cleaning is performed when the robot is immobilized into its max-span pose - all suction cups are $\mathrm{ON}$ and balancing jets are activated. This provides the center of robot's mass close to the surface, which means maximum stability of the robot on the surface.

Changing orientation (rotation around $Z$-axis): While crawling is implemented by rotation around $Y$-axis, the changing of robot orientation is done by rotation around $Z$-axis. It can be performed around the center of any suction cup, meaning that there are four possible centers of rotation. Similar approach of applying a control sequence of activating/deactivating motor M2 and the suction cups is used. The sequence of rotating clockwise around suction cup A is illustrated in Fig.2. Similarly to crawling, during change of orientation the balancing jets are activated when any suction cup is OFF, as they all have passive DOFs around $\mathcal{R}_{Z}$. 
Stepping forward (on $Y$-axis): Due to the non-holonomic properties of the robot construction, direct stepping forward on $Y$-axis cannot be performed. This is achieved by applying the same strategy of changing direction. In fact, we can do it as a consecutive execution of four rotations around $Z$-axis - around cup $\mathbf{A}$ and around cup $\mathbf{C}$ as shown on Fig.2.

\section{Robot stability on the surface}

In this study we discuss two typical cases when the crawling robot loses stability on a inclined surface - tumbling and sliding. Definitely, any of these two cases disrupts the normal motion and the robot control system should apply a compensating action. Obviously, the system must respond to the appearance of the the first event of sliding or tumbling. In this section, we are going to investigate the condition of these cases individually to assess the global robot stability.

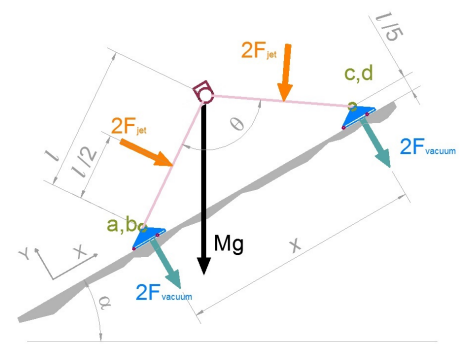

Fig. 3. Robot simplified model

For the analysis, we consider a simplified model as shown on Fig. 3. Here, we assume that the weight of the robot is $M g$, friction between the suction cup and surface is $\mu$, adhesion force of one suction cup is $F_{\text {vacuum }}$, and balancing force of one jet is $F_{j e t}$. We want to see what is the minimum adhesion force to keep the robot on the surface only by means of suction cups without using the balancing jets,and the influence of the inclination angle $\alpha$ and the crawling angle $\theta$ (Fig. 3) on this force.

\subsection{Robot stability in stationary phase}

Tumbling appears also when the adhesion is not enough to keep the suction cups on the surface, but at lease one is still attached to the surface. Let us assume that an instant tumbling motion occurs around lower axle a-b. The moment generated by the adhesion force $2 F_{\text {vacuum }}$ of suction cups $\mathbf{C}$ and $\mathbf{D}$ should be larger than the moment generated by the weight $M g$ of 
the robot to maintain stable surface contact and not to tumble:

$$
2 F_{\text {vacuum }} \cdot 2 l \geqslant-M g \cdot l \cdot \cos \alpha \cdot \sin \frac{\theta}{2}+M g \cdot l \cdot|\sin \alpha| \cdot\left(p+\cos \frac{\theta}{2}\right),
$$

where $p$ is a parameter giving the height of the suction cup joint over the contact surface. On Fig. 3 it is $p=\frac{l}{5}$ according to the construction of our prototype. It was convenient to introduce this parameter for simplicity, then the required adhesion force $F_{\text {vacuum }}$ of each suction cup is:

$$
F_{\text {vacuum }} \geqslant \frac{M g}{4} \cdot\left(|\sin \alpha| \cdot\left(p+\cos \frac{\theta}{2}\right)-\cos \alpha \cdot \sin \frac{\theta}{2}\right),
$$

Fig. 4 shows the adhesion force $F_{\text {vacuum }}$ as a function of the crawling angle $60^{\circ} \leqslant \theta \leqslant 180^{\circ}$, and the inclination angle $-180^{\circ} \leqslant \alpha \leqslant 180^{\circ}$ we have a

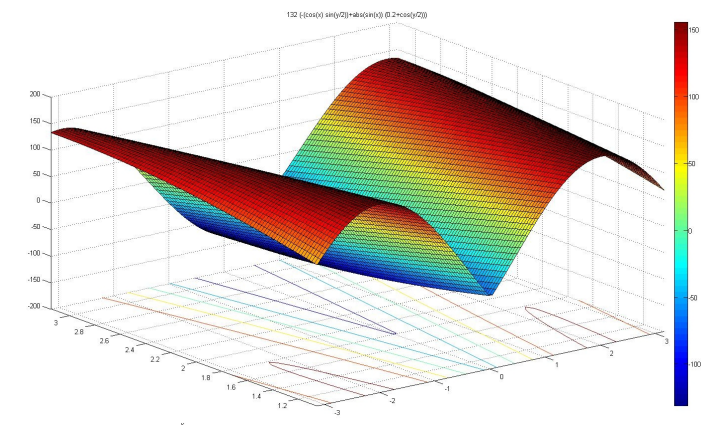

Fig. 4. Required adhesion to prevent tumbling - all cups are ON and M1 is OFF

minimum at $\alpha=0^{\circ}$ and we notice that the crawling angle changes the position of the two maximums. The effect of $\alpha$ on the value of these maximums is not so important, so we can take the higher value as a required adhesion force for these maximums.

Sliding appears if the adhesion is not enough to keep all suction cups on the surface, such that external forces having orthogonal projection against the adhesion force can move the robot or slide it on the surface.

Following the same reasoning, stability against sliding of the robot can be provided if the friction generated by the negative pressure adhesion force by all four suction cups is larger than the force generated by its weight:

$$
\mu\left(4 F_{\text {vacuum }}-M g|\cos \alpha|\right) \geqslant M g \sin \alpha,
$$

yielding the required adhesion force $F_{\text {vacuum }}$ of each cup to prevent sliding:

$$
F_{\text {vacuum }} \geqslant \frac{M g}{4}\left(\frac{\sin \alpha}{\mu}+|\cos \alpha|\right)
$$


Clearly, the required force $F_{\text {vacuum }}$ to prevent sliding is independent of crawling angle $\theta$. Fig. 5 shows the adhesion force $F_{\text {vacuum }}$ as function of $\alpha$.

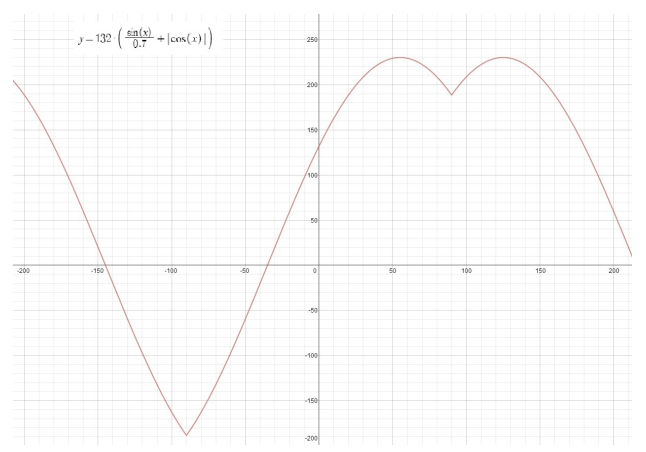

Fig. 5. Required adhesion prevent sliding - all suction cups are ON and M1 is OFF

From Fig. 5 and Fig. 4 we can get the needed force to maintain stable adhesion against sliding and tumbling. Clearly, the maximum of $F_{\text {vacuum }}$ for non sliding is greater than all the maxima in the non tumbling case.

\subsection{Balancing force during crawling (motion phase)}

When the robot start crawling, a pair of suction cups is OFF (according to Fig. 3 cups $\mathrm{A}$ and $\mathrm{B}$ are $\mathrm{OFF}$ ) and the balancing water jet apply a force to maintain the adhesion on the surface during this movement. We analyze this case in similar way as in Section 3.1.

The moment generated by the balancing jets $2 F_{\text {jet }}$ should be larger than the moment generated by the weight $M g$ of the robot to maintain stable surface contact and not to tumble:

$$
2 F_{j e t} \cdot(1-\cos \theta) \geqslant-M g \cdot l \cdot \cos \alpha \cdot \sin \frac{\theta}{2}+M g \cdot l \cdot|\sin \alpha| \cdot\left(p+\cos \frac{\theta}{2}\right),
$$

yielding the required force for each balancing jet $F_{j e t}$ :

$$
F_{j e t} \geqslant \frac{M g l}{2} \cdot \frac{\left(|\sin \alpha| \cdot\left(p+\cos \frac{\theta}{2}\right)-\cos \alpha \cdot \sin \frac{\theta}{2}\right)}{1-\cos \theta}
$$

Fig. 6 shows the balancing force $F_{\text {jet }}$ needed to keep the adhesion to the surface during the crawling for crawling angle $60^{\circ} \leqslant \theta \leqslant 180^{\circ}$, and the inclination angle $-180^{\circ} \leqslant \alpha \leqslant 180^{\circ}$. There is local minimum for $\alpha=0^{\circ}$ and the smaller the crawling angle the greater the two maxima are, that means in the control process when $\alpha$ is around $+90^{\circ}$ or $-90^{\circ}$, it is better not to keep $80^{\circ} \leqslant \theta \leqslant 180^{\circ}$, so the maximum of $F_{j e t}$ will be smaller. 


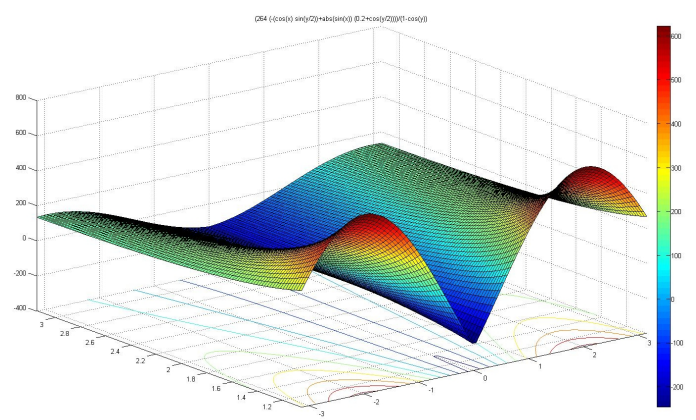

Fig. 6. Required water jet force for adhesion on the surface while the robot is crawling

\subsection{Torque of M1 during crawling (motion phase)}

The required torque for crawling is calculated by applying Newton's second law $\sum \vec{\Gamma}=J \vec{\gamma}$, assuming the angular velocity of $\mathbf{M 1}$ is constant then $\vec{\gamma}=\overrightarrow{0}$, we obtain:

$T-M g \cdot l \sin \left(\frac{\theta}{2}-\alpha\right)-2 F_{j e t} \cdot l(1-\cos \theta)-\mu \cdot p l\left(4 F_{j e t} \sin \frac{\theta}{2}+M g \cdot|\cos \alpha|\right)=0$, and the torque of $\mathbf{M 1}$ during the crawling :

$$
T=M g \cdot l\left(\sin \left(\frac{\theta}{2}-\alpha\right)+\mu p|\cos \alpha|\right)+2 F_{j e t} \cdot l\left(1-\cos \theta+\mu \cdot p \sin \frac{\theta}{2}\right),
$$

Substituting $F_{\text {jet }}$ we obtain the result shown on Fig. 7 , which shows the torque $T$ needed during the crawling for $60^{\circ} \leqslant \theta \leqslant 180^{\circ}$, and $-180^{\circ} \leqslant \alpha \leqslant$ $180^{\circ}$. It is clear that we need more torque when $\alpha \leqslant 0^{\circ}$ and the movement against the gravity, and in these cases the smaller $\theta$ the greater the required torque, so either motor M1 should bear these loads or the control process must avoid these cases and crawl with $\theta \geqslant 90^{\circ}$ or more.

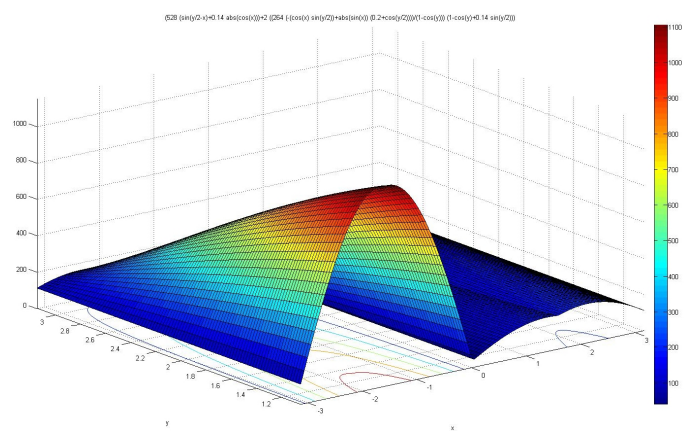

Fig. 7. Required torque from motor M1 during robot crawling 


\section{Conclusion}

A new concept of crawling robot for in-water cleaning of surfaces is presented in this paper. The design is based on minimalistic approach to achieve a compromise between maximum performance and reasonable complexity and cost of the system.

The solution is characterized by using minimum active DOFs (only two) for performing movements practically in all possible directions on a nonflat $2 \mathrm{D}$ surface. The available passive DOFs provide adaptability to local concavity/convexity of the surface to be cleaned.

Different types of unstable conditions related to the inclination of the surface and the crawling angle are considered and discussed for keeping contact with the surface in different situations. This stability study helps us in the future to estimate the adhesion force of the suction cups, also in the control of the needed balancing forces while the suction cups are in OFF-state as a function of the measured surface inclination angle and the robot pose to keep the robot stability.

\section{References}

1. H. Albitar, A. Ananiev and I. Kalaykov, New concept of in-water surface cleaning robot, in Mechatronics and Automation (ICMA), 2013 IEEE International Conference on, 2013.

2. D. Sun, J. Zhu and S. K. Tso, Climbing 8 walking robots towards new applications , p. 546 (2007).

3. X.-H. Li, Y.-Q. Zhu, G.-Q. Huang and S.-L. Nie, Journal of Chongqing University (English Edition). China 8, 63 (2009).

4. G.-q. Huang, Y.-s. Yang, X.-h. Li and Y.-q. Zhu, Journal of Shanghai University (English Edition) 13, 305 (2009).

5. S. Guo, X. Lin and S. Hata, A conceptual design of vectored water-jet propulsion system, in Mechatronics and Automation, 2009. ICMA 2009. International Conference on, 2009. 